\title{
ON THE CLASSIFICATION OF THE MAPPINGS OF A 2-COMPLEX
}

\author{
BY \\ HERBERT ROBBINS
}

1. Introduction. The continuous mappings of one complex into another may be classified in two essentially different ways. Two mappings are called homotopic if one may be deformed into the other, and the relation of homotopy partitions the set of all mappings of a complex $K$ into a complex $T$ into disjoint subsets of homotopic mappings. This classification is possible for the most general spaces, irrespective of their combinatorial structure. The other way of classifying mappings is according to their homology behavior. Any mapping $f$ of $K$ into $T$ may be deformed into a simplicial mapping, assigning to the vertices of each simplex of $K$ the vertices of a simplex of $T$ of the same or lower dimension, and affine in each simplex $\left({ }^{1}\right)$. Such a mapping induces a homomorphism of the homology groups of $K$ into those of $T$, and of the cohomology groups of $T$ into those of $K$, for each dimension $r$ and coefficient group $G$. Two mappings which induce the same homomorphisms for each $r$ and $G$ may be called homologous, and the set of mappings of $K$ into $T$ may be classified by this relation. It is easy to show that if two mappings are homotopic they are homologous. The converse is not true in general, but holds if $K$ is an $n$-complex and $T$ an $n$-sphere. This theorem is due to H. Hopf, and has been generalized by W. Hurewicz to the case where $T$ is one of a more general class of spaces whose $r$ th homotopy groups vanish for $r<n\left({ }^{2}\right)$.

Mappings may also be classified according to the homomorphisms they induce of the fundamental group of $K$ into that of $T$. Again, homotopic mappings induce the same homomorphisms. Under certain conditions, when $T$ is an "aspherical" space, whose $r$ th homotopy groups vanish for $r>1$, these classifications coincide, as shown by Brouwer and Hurewicz $\left({ }^{3}\right)$.

Although in general homotopy provides a more finely graduated classification of mappings than combinatorial and group-theoretical methods, it is one

Presented to the Society, September 6, 1938; received by the editors May 25, 1940. This paper contains the results of a doctoral dissertation accepted by Harvard University in May, 1938.

(1) See Alexandroff-Hopf, Topologie, pp. 314-319.

(2) See Hopf, Commentarii Mathematici Helvetici, vol. 5 (1932), pp. 39-54, also AH, chap. 13, and Hurewicz, Proceedings, Akademie van Wetenschappen, Amsterdam, vols. 38-39 (1935-1936); in particular, vol. 39, pp. 117-126.

(3) See Brouwer, Mathematische Annalen, vol. 82 (1921), Hopf, Journal für die reine und angewandte Mathematik, vol. 165 (1931), pp. 225-236, and Hurewicz, loc. cit., vol. 39, pp. 215222 . 
of the objects of combinatorial topology to give combinatorial conditions which will be sufficiently strong to ensure homotopy. In all cases where such conditions have been given heretofore, with the exception of some recent work by $\mathrm{H}$. Whitney $\left({ }^{4}\right)$, the space $T$ is assumed to be simply-connected, or the higher dimensional homotopy groups are assumed to vanish. It is the object of this paper to give combinatorial methods which shall be adequate to classify the mappings of a 2-complex $K=K^{2}$ into a perfectly general space $T$. The results of the present paper give necessary and sufficient conditions for the homotopy of two mappings of a 2-complex into a space $T$; these involve the use of chains in $K$ with coefficients from the fundamental group and 2-dim. homotopy group of $T$, and are given in Theorem 4 .

The author wishes to express his deep indebtedness to the work of Whitney mentioned above for the stimulation of his interest in this subject. Without the help of Professor Whitney's many suggestions and constant counsel and encouragement this paper could not have been written.

2. Coboundary and product of chains. By the coboundary $\delta \sigma^{n}$ of an $n$-simplex $\sigma^{n}=x_{0} x_{1} \cdots x_{n}$ we mean the set of all $(n+1)$-simplexes which have it as an $n$-face, with orientations determined by $\delta x_{0} x_{1} \cdots x_{n}=\sum x x_{0} x_{1} \cdots x_{n}$ where the summation is over all vertices $x$ of $K$ such that $x x_{0} x_{1} \cdots x_{n}$ is an $(n+1)$-simplex of $K\left(^{5}\right)$. A chain is a cocycle if its coboundary vanishes, and two cocycles are cohomologous if their difference is a coboundary.

Let the vertices of the complex $K$ be given in a definite order, and suppose that $x_{i} x_{j} \cdots x_{l}$ is positively oriented when $i<j<\cdots<l$. Let $A^{r}=\sum \alpha_{i_{0}} \cdots_{i_{r}} x_{i_{0}} \cdots x_{i_{r}}$ and $B^{s}=\sum \beta_{i_{0}} \cdots_{i_{s}} x_{i_{0}} \cdots x_{i_{s}}$ be $r$ - and $s$-chains respectively. Then by their product $A^{r} \cup B^{s}$ we mean the $(r+s)$-chain $\left(^{6}\right)$

$$
A^{r} \cup B^{s}=\sum \alpha_{i_{0}} \cdots i_{r} \beta_{i_{r}} \cdots i_{r+s} x_{i_{0}} \cdots x_{i_{r+s}} .
$$

The coefficients $\alpha$ and $\beta$ may be from a group or from any system for which an operation $\alpha \beta$ is defined.

3. The space $T$ and its covering space. Let $P$ be a fixed point of the space $T\left({ }^{7}\right)$. The paths $P p$ from $P$ to the points $p$ of $T$ define the points of the covering space $\bar{T}$. Two paths are equivalent if they have the same endpoint and if one may be deformed into the other leaving the endpoints fixed; equivalent paths define the same point of $\bar{T}$, which is topologized in the usual way, by letting a neighborhood of a path consist of all paths which are continuations

(4) Unpublished; classifying mappings into projective spaces. Many of the methods used in this paper have been used in the present work. See also S. Eilenberg, Annals of Mathematics, vol. 41 (194) i, pp. 231-251, for references to work of Pontrjagin and Freudenthal.

(5) See Whitney, three papers in Duke Mathematical Journal, vol. 3 (1937); especially pp. 51-55.

${ }^{(6)}$ See Whitney, Proceedings of the National Academy of Sciences, vol. 23 (1937), pp. 285291 ; especially p. 286.

(7) It is usual to assume that $T$ is connected and locally 0 - and 1-connected. 
of it within a given neighborhood of its endpoint. We shall denote by $\Phi$ the function which assigns to each point of $\bar{T}$ the endpoint of a path which defines it; then $\Phi$ maps $\bar{T}$ onto $T$. A path (or rather, a class of equivalent paths) with endpoint $p$ will be said to lie over the point $p$, and may be denoted by $p_{i}$, the subscript denoting the particular path from $P$ to $p$ which defines it. We denote the "point-path" which lies over $P$ by $\bar{P}$.

Let $S$ be a simply-connected space (that is, one whose fundamental group vanishes) with a fixed point $s$, and let $f$ map $S$ into $T$ with $f(s)=P$. Then there is a mapping $\bar{f}$ of $S$ into $\bar{T}$ with $\bar{f}(s)=\bar{P}$, defined as follows: choose a path $s x$ in $S$ from $s$ to $x$, and let $\bar{f}(x)$ be the path in $T$ (i.e., point of $\bar{T}$ ) defined by $f(s x)$. Since $S$ is simply-connected, this is independent of the particular path chosen. We call $\bar{f}$ the mapping induced by $f$. Clearly, $\Phi \bar{f}=f$. Conversely, let $F$ be a mapping of $S$ in to $\bar{T}$ with $F(s)=\bar{P}$; then $f=\Phi F$ is a mapping of $S$ into $T$ with $f(s)=P$ such that $\bar{f}=F$. Let $f_{1}$ and $f_{2}$ be two mappings of $S$ into $T$, with $f_{1}(s)=f_{2}(s)=P$; then $f_{1}$ and $f_{2}$ may be deformed into each other, keeping $s$ at $P$, if and only if $\bar{f}_{1}$ and $\bar{f}_{2}$ may be deformed into each other, keeping $s$ at $\bar{P}$. Thus the homotopy-classes of the mappings of $S$ into $T$ are put in to one-toone correspondence with those of $S$ into $\bar{T}$, with $s$ at $P$ or $\bar{P}$ respectively.

Let $S_{0}^{2}$ be the unit 2-sphere in Euclidean 3-space, with a south pole $P_{0}$. The homotopy-classes of $S_{0}^{2}$ in to $T$ with $P_{0}$ going into $P$ are the elements of the 2-dimensional homotopy group $\pi_{2}(T)\left({ }^{8}\right)$. By pushing away from $P_{0}$ it is clear that each element of $\pi_{2}(T)$ may be represented by a mapping in which each point of $S_{0}^{2}$ goes in to $P$, with the exception of a small 2-cell whose boundary goes into $P$ and whose interior is mapped into $T$ as though it were a 2-sphere corresponding to $S_{0}^{2}$. Clearly, this patch may be pushed over $S_{0}^{2}$ into any position. If the element $h$ of $\pi_{2}(T)$ is defined by the mapping $f$, we write $h=h_{f}$. The sum $h_{f_{1}}+h_{f_{2}}$ of two elements of $\pi_{2}(T)$ is defined by a mapping of $S_{0}^{2}$ in to $T$ which carries each point of $S_{0}^{2}$ in to $P$, except for two disjoint patches, one of which is mapped by $f_{1}$ and the other by $f_{2}$. Since the position of the patches is of no consequence, $\pi_{2}(T)$ is seen to be an abelian group. By the preceding paragraph, $\pi_{2}(T) \approx \pi_{2}(\bar{T})$, where the isomorphism is defined by letting each mapping $f$ correspond to its induced mapping $\bar{f}$.

Let $G$ be the fundamental group of $T$, with unit element 1 . We shall define an operation $g h$, where $g$ is any element of $G$ and $h$ is in $\pi_{2}(T)$, such that $g h$ is in $\pi_{2}(T)$ and the laws

$$
\begin{aligned}
g_{1}\left(g_{2} h\right) & =\left(g_{1} g_{2}\right) h, \\
1 h & =h, \\
g\left(h_{1}+h_{2}\right) & =g h_{1}+g h_{2}
\end{aligned}
$$

$\left.{ }^{8}\right)$ For homotopy groups, see Hurewicz, loc. cit. $S_{0}^{2}$ may be replaced by any homeomorphic set, with a fixed point and a chosen orientation. 
hold. The definition of $g h_{f}$ is as follows: let a circle rise from $P_{0}$ to the equator as $t$ goes from 0 to 1 ; map this 1 -cell of circles into the points of a path $\Gamma$ in $T$ defining $g$, each circle $C_{t}$ going into the corresponding point $\Gamma_{t}$ of $T$, and map the upper hemisphere of $S_{0}^{2}$ into $T$ by $f$. The resulting mapping defines $g h_{f}$, and the three properties stated are easily verified.

If a fixed mapping $f$ defining $h_{f}$ of $\pi_{2}(T)$ carries another point $P^{*}$ into $P$ also, we may regard $P^{*}$ as the fixed point of $S_{0}^{2}$ and get another element $h_{f^{*}}$. More precisely, we may consider the mapping $f^{*}$ of $S_{0}^{2}$ in to $T$ obtained by first rotating $S_{0}^{2}$ to bring $P^{*}$ to $P_{0}$ and then mapping by $f$. Suppose $f$ maps a path $P_{0} P^{*}$ into $g$ of $G$. Then

$$
h_{f}^{*}=g^{-1} h_{f} .
$$

This is clear if we consider the induced mappings $\bar{f}$ and $\bar{f}^{*}$ into $\bar{T}$. For under $\bar{f}$, a point $x$ of $S_{0}^{2}$ is mapped into a path $f\left(P_{0} x\right)$, while under $\bar{f}^{*}$ it is mapped into a path which is equivalent to the path $g^{-1} f\left(P_{0} x\right)$. This fact may be used to give a simple geometrical proof of (3.3).

Suppose $f$ maps $S_{0}^{2}$ into $T$, defining the element $h$ of $\pi_{2}(T)$, in the following manner: a small 2-cell $\sigma$ goes in to $P$, and a path $P_{0} P^{*}$ from $P_{0}$ to the boundary of $\sigma$ goes into the element $g$ of $G$. Then if we replace the mapping of $\sigma$ by one which (regarding $\sigma$ as a 2-sphere) defines the element $h^{\prime}$ of $\pi_{2}(T)$, the resulting mapping of the whole of $S_{0}^{2}$ defines an element $H$ of $\pi_{2}(T)$, and

$$
H=h+g h^{\prime} \text {. }
$$

For regarding $P^{*}$ as the fixed point of $S_{0}^{2}$ shows that $g^{-1} H=g^{-1} h+h^{\prime}$ by definition of the sum of two elements of $\pi_{2}(T)$, and going back to $P_{0}$ (i.e., applying g) gives the desired equality.

We shall have occasion to regard the boundary $\partial E^{3}$ of a 3-cell $E^{3}$ as a 2 -sphere, and a mapping of $\partial E^{3}$ as defining an element of $\pi_{2}(T)$. This is completely specified as soon as we have chosen a particular point of $\partial E^{3}$ as the fixed point; the particular homeomorphism of $\partial E^{3}$ with $S_{0}^{2}$ which defines the element of $\pi_{2}(T)$ may be set up by placing the 3-cell inside $S_{0}^{2}$ and projecting from the center of $S_{0}^{2}$ so that the point chosen as the fixed point of $\partial E^{3}$ goes into $P_{0}$. The precise manner in which this is done is immaterial, since we are concerned only with homotopy invariants.

Suppose $E_{1}^{3}=x_{0} x_{1} x_{2} x_{3}$ and $E_{2}^{3}=y_{0} y_{1} y_{2} y_{3}$ are two 3 -cells whose boundaries are regarded as 2 -spheres with fixed points $x_{0}$ and $y_{0}$ respectively, and suppose $f_{1}$ and $f_{2} \operatorname{map} E_{1}^{3}$ and $E_{2}^{3}$ into $T$ with $f_{1}\left(x_{0}\right)=f_{2}\left(y_{0}\right)=P$. Then two elements $h_{f_{1}}$ and $h_{f_{2}}$ of $\pi_{2}(T)$ are defined. Now suppose that the 2-cells $x_{0} x_{1} x_{2}$ and $y_{0} y_{1} y_{2}$ are congruent, with $x_{i}$ corresponding to $y_{i}$, and that $f_{1}$ on $x_{0} x_{1} x_{2}$ coincides with $f_{2}$ on $y_{0} y_{1} y_{2}$ under this congruence. Then we may form a 2 -sphere with fixed point $x_{0}$ by placing $E_{1}^{3}$ and $E_{2}^{3}$ together, bringing $x_{i}$ into coincidence with $y_{i}$ $(i=0,1,2)$ and dropping out the 2-cells $x_{0} x_{1} x_{2}$ and $y_{0} y_{1} y_{2}$. Denoting the element of $\pi_{2}(T)$ thus defined by $h$, we have $\left({ }^{9}\right)$ 


$$
h=h_{f_{1}}+h_{f_{2}} .
$$

For $h_{f_{1}}+h_{f_{2}}$ is defined by a mapping homotopic to that defined by $h$, except that there are two 2-cells adjacent along an edge, and such that the mapping of one is the reflection of the mapping of the other in this edge. This patch may be eliminated by a simple deformation, which proves the above relation. (3.6) also holds if the 3 -simplexes $E_{i}^{3}$ are replaced by 3 -cells, with mappings coinciding along congruent 2 -faces.

Let $G$ be a group (not necessarily abelian) with elements $g$ and operation $g_{1} g_{2}=g_{3}$. By the group ring $\bar{G}$ we mean the set of linear forms $\sum a_{i} g_{i}$, where the $a_{i}$ are integers, and only a finite number of terms appear. We assume the laws

$$
\begin{gathered}
(a+b) g=a g+b g, \\
a(b g)=(a b) g .
\end{gathered}
$$

Then $\bar{G}$ is a ring, where + is defined as formal addition, and · by the law $\left({ }^{10}\right)$

$$
\sum a_{i} g_{i} \cdot \sum b_{i} g_{i}^{*}=\sum\left(a_{i} b_{j}\right) g_{i} g_{j}^{*} .
$$

We now assume that $G$ operates on the abelian group $H$, i.e., that a multiplication $g h$ is defined, where $g h$ is in $H$, and the laws (3.1) to (3.3) are satisfied. If we now define

$$
\left(\sum a_{i} g_{i}\right) h=\sum a_{i}\left(g_{i} h\right),
$$

then $\bar{G}$ will be a ring of operators on $H$, the laws (3.1) to (3.3) remaining valid. In future applications, $G$ will be the fundamental group of $T$ and $H$ will be $\pi_{2}(T)$.

Let the fundamental group $G$ of $T$ be given by a set of generators $g_{1}, g_{2}, \ldots$ and a set of relations $R_{1}, R_{2}, \cdots$. Any product of elements of $G$ may be called a word, and any word may be written as a product $g_{1}^{\alpha_{1}} g_{2}^{\alpha_{2}} \cdots g_{p}^{\alpha_{p}}$ of the generators. For each element $g$ of $G$ we choose a fixed representation as a product of the $g_{i}$, and call this the normal form for $g$. Any product of the $g_{i}$ which equals 1 may be shown to do so by using a succession of the relations $R_{i}$, together with the trivial relations $g_{i} g_{i}^{-1}=g_{i}^{-1} g_{i}=1$; for each product we choose a definite manner of doing so. For each generator $g_{i}$ we choose a definite path $\sigma_{i}$ in $T$ determining it; if $g=g_{1}^{\alpha_{1}} \cdots g_{p}^{\alpha_{p}}$ in normal form, we choose as the definite path determining it the path $\sigma_{1}^{\alpha_{1}} \cdots \sigma_{p}^{\alpha_{p}}$. Then each relation says that a certain path may be shrunk to a point. If the path is $\theta=\sigma_{1}^{\alpha_{1}} \cdots \sigma_{p}^{\alpha_{p}}$, then if the 1-sphere $S^{\prime}$ is mapped over $\theta$, this mapping may be extended throughout the interior $R^{2}-S^{\prime}$ of $S^{\prime}$. We choose a definite manner of doing this; then to each $R_{j}$ corresponds a mapping $\phi_{j}$ of $R^{2}$ in to $T$, defining the (singular) 2-chain

(9) We suppose the 2-spheres are oriented like $\partial\left(x_{0} x_{1} x_{2} x_{3}\right),-\partial\left(y_{0} y_{1} y_{2} y_{3}\right)$, and $\partial\left(x_{0} x_{1} x_{2} x_{3}\right.$ $\left.-y_{0} y_{1} y_{2} y_{3}\right)$.

${ }^{\left({ }^{10}\right)}$ See K. Reidemeister, Mathematische Annalen, vol. 112 (1936) for a similar use of $\bar{G}$. 
$C_{j}=\phi_{j}\left(R^{2}\right)$ of $T$, with boundary $\sum \alpha_{i} \sigma_{i}$. Likewise there exist the induced mappings $\bar{\phi}_{j}$ of $R^{2}$ in to $\bar{T}$, and the chains $\bar{C}_{j}=\bar{\phi}_{j}\left(R^{2}\right)$.

4. The complex $K$; standard mappings. Let $K$ be a connected, simplicial complex with vertices $x_{i}$ which are ordered in a definite manner according to their subscripts: $x_{1}<x_{2}<x_{3}<\cdots$. We assume that the simplexes $x_{i} x_{j} \cdots x_{l}$ are positively oriented if $i<j<\ldots<l$.

Let $f$ map $K$ into $T$. We call $f$ normal over $K^{1}$-the 1 -dim. part of $K$-if it maps each vertex $x_{i}$ of $K$ into the fixed point $P$ of $T$, and each 1-cell $x_{i} x_{j}$ into an element $\sigma_{1}^{\alpha_{1}} \cdots \sigma_{r}^{\alpha_{r}}$ of $G$ in normal form, with the convention that the normal form for the path defining the unit element of $G$ is the "point-path" $P$.

Lemma 1. Any mapping $f$ of $K$ into $T$ may be deformed into a mapping normal over $K^{1}$.

The required deformation is obtained by first bringing all the vertices to $P$, then extending the deformation through the rest of $K$, then deforming the mapping so that all the 1-cells are in normal form and extending the deformation through $K$, using a simple lemma on such extensions( $\left.{ }^{11}\right)$.

Let $f$ be a mapping of $K$ into $T$, normal over $K^{1}$. Then we may define a mapping $f^{0}$ of $K^{\prime \prime}\left({ }^{12}\right)$ into $T$, coinciding with $f$ on $K^{1}$, and defined in the 2-cells of $K$ as follows: running around the boundary of a 2-cell $\sigma^{2}$ of $K$ defines three elements of $G$, each in normal form; there is a corresponding definite deformation of their product to $P$ (since this is equivalent to the existence of an extension of the mapping defined on the boundary of $\sigma^{2}$ throughout its interior) using the $R_{i}$, and corresponding mappings into $T$. Thus we define $f^{0}$ throughout the interior of $\sigma^{2}$, giving $f^{0}\left(\sigma^{2}\right)=\sum \alpha_{i} C_{i}$. A mapping $f$ which coincides with the mapping $f^{0}$ thus determined throughout a 2 -cell $\sigma^{2}$ we shall call standard over $\sigma^{2}$; a mapping which is standard over each 2-cell of $K$ we shall call standard (note that if $\sigma=x_{i} x_{j} x_{k}, i<j<k$, the three elements of $g$ are, in order, $g\left(x_{i} x_{j}\right), g\left(x_{j} x_{k}\right)$, and $g^{-1}\left(x_{i} x_{k}\right)$.)

5. An example. In this section we shall illustrate the concepts defined in the preceding pages by choosing a particular space $T=$ "torus with patch," defined as follows: choose a definite simplicial subdivision of the torus, and adjoin to it another 2 -simplex $A$ whose boundary only is identified with that of a congruent 2-simplex of the torus. Intuitively, this space corresponds to an inflated inner tube with a small patch cemented to the tube around its edge. The fundamental group $G$ of this space has two generators, $g_{1}$ and $g_{2}$, with the single relation

$$
g_{1} g_{2}=g_{2} g_{1} \text {. }
$$

The elements of $G$ may be represented by ordered pairs of integers $\left(\alpha_{1}, \alpha_{2}\right)$, with the law of addition given by $\left(\alpha_{1}, \alpha_{2}\right)=\left(\beta_{1}, \beta_{2}\right)=\left(\alpha_{1}+\beta_{1}, \alpha_{2}+\beta_{2}\right)$.

(11) See Whitney, loc. cit., in note 5, pp. 52-53.

(12) $K^{\prime \prime}$ is the 2-dim. part of $K$. 
We choose paths $\sigma_{i}$ defining the $g_{i}$ as two circles on the torus; then corresponding to the relation $(R)$ we have the fact that if the boundary of a 2-cell $\sigma^{2}$ be mapped into the path $\sigma_{1}+\sigma_{2}-\sigma_{1}-\sigma_{2}$, this mapping may be extended throughout the interior of $\sigma^{2}$. It is natural to choose as the corresponding definite mapping of the interior of the 2-cell into $T$ the mapping into all of $T$ except the patch; i.e., into $T-A$, as though $T$ were simply a torus.

Each element of $G$ is of the form $\left(\alpha_{1}, \alpha_{2}\right)$, where the $\alpha_{i}$ are positive, negative, or zero; we choose as the normal form for such an element the path which
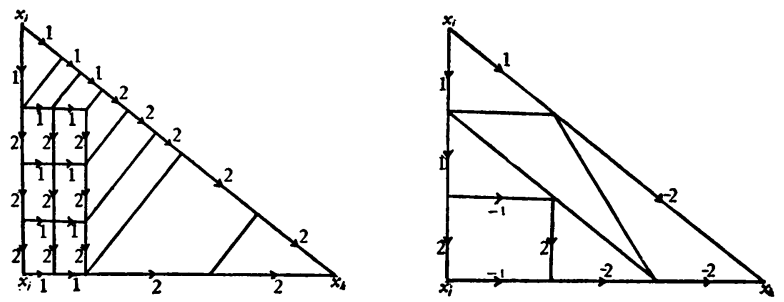

FIG. 1

first runs around $\sigma_{1} \alpha_{1}$ times, then around $\sigma_{2} \alpha_{2}$ times (in the positive or negative senses, according to the signs of the $\alpha_{i}$ ).

If $f$ is a normal mapping of the boundary of a 2-cell $x_{i} x_{j} x_{k}$ into $T$ for which

$$
g\left(x_{i} x_{j}\right)=\left(\alpha_{1}, \alpha_{2}\right), \quad g\left(x_{j} x_{k}\right)=\left(\beta_{1}, \beta_{2}\right), \quad g\left(x_{i} x_{k}\right)=\left(\gamma_{1}, \gamma_{2}\right)
$$

are the elements of $G$ into which the 1-cells of $x_{i} x_{j} x_{k}$ are mapped by $f$, then the necessary and sufficient condition that $f$ may be extended throughout the interior of $x_{i} x_{j} x_{k}$ is that

$$
\alpha_{1}+\beta_{1}=\gamma_{1}, \quad \alpha_{2}+\beta_{2}=\gamma_{2} .
$$

(This will be the case if $f$ is derived from any mapping of $K$ into $T$ normal over $K^{1}$, and $x_{i} x_{j} x_{k}$ is a 2-cell of $K$.) As our definite manner of deforming the boundary of $x_{i} x_{j} x_{k}$ to $P$, we choose the following sequence of equalities in $G$, using the trivial relations and $(\mathrm{R})$ :

$$
\begin{aligned}
\alpha_{1} \sigma_{1}+\alpha_{2} \sigma_{2}+\beta_{1} \sigma_{1}+\beta_{2} \sigma_{2} & =\alpha_{1} \sigma_{1}+\left(\alpha_{2}-1\right) \sigma_{2}+\sigma_{2}+\sigma_{1}+\left(\beta_{1}-1\right) \sigma_{1}+\beta_{2} \sigma_{2} \\
& \rightarrow \alpha_{1} \sigma_{1}+\left(\alpha_{2}-1\right) \sigma_{2}+\sigma_{1}+\sigma_{2}+\left(\beta_{1}-1\right) \sigma_{1}+\beta_{2} \sigma_{2} \\
& \rightarrow \cdots \rightarrow\left(\alpha_{1}+\beta_{1}\right) \sigma_{1}+\left(\alpha_{2}+\beta_{2}\right) \sigma_{2}=\gamma_{1} \sigma_{1}+\gamma_{2} \sigma_{2} .
\end{aligned}
$$

In Figure 1 we indicate the corresponding mappings of the interior of $x_{i} x_{j} x_{k}$ in to $T$ for two cases:

$\left(\alpha_{1}, \alpha_{2}\right)=(1,3), \quad\left(\beta_{1}, \beta_{2}\right)=(2,2) ; \quad\left(\alpha_{1}, \alpha_{2}\right)=(2,1), \quad\left(\beta_{1}, \beta_{2}\right)=(-1,-2)$.

The general situation is then clear. Note that in the resulting subdivision of $x_{i} x_{j} x_{k}$, each small 2-cell is mapped either into $\pm \sigma_{i}$ or into $T-A$, but in no 
case does a point of $x_{i} x_{j} x_{k}$ lie in $A$. This gives the definition of standard mapping for this example.

The covering space $\bar{T}$ of $T$ is the plane, ruled off in to congruent rectangles as in the case of the torus alone, except that here each rectangle has a small patch like that of $T$. We may number each rectangle with a double subscript, and denote the parts of each rectangle by the symbol denoting the corresponding part of $T$, with a bar above and a double subscript:

$$
\bar{T}_{i j} \rightarrow T, \quad \bar{A}_{i j} \rightarrow A, \quad \bar{P}_{i j} \rightarrow P\left({ }^{13}\right) .
$$

The nature of $\pi_{2}(T)$ follows from the

LeMma 2. Let $W$ be a simply-connected space with fixed point $P$, and let $S^{2}$ be a 2-sphere. By $W+S^{2}$ we mean the space obtained by identifying a single point of $S^{2}$ with $P$. Then $\pi_{2}\left(W+S^{2}\right)$ is isomorphic with $\pi_{2}(W)+I_{0}$, where $I_{0}$ is the additive group of integers.

Proof. Let $f$ be a mapping of the 2 -sphere $S_{0}^{2}$ subdivided simplicially into $W+S^{2}$ with $f\left(P_{0}\right)=P$. The subdivision may be chosen so that each 2 -cell $\sigma_{i}^{2}$ of $S_{0}^{2}$ is mapped into either $W$ or $S^{2}$ but not both. We may deform $f$ so that all vertices of $S_{0}^{2}$ lie at $P$, and since $W+S^{2}$ is simply-connected, we may further $\operatorname{deform} f$ so that all the 1-cells of $S_{0}^{2}$ lie at $P$. A further deformation will now be made. Consider any 2-cell $\sigma^{2}$ of $S_{0}^{2}$; its boundary is mapped into $P$. We $\operatorname{deform} f$ in $\sigma^{2}$ by shrinking $\sigma^{2}$ into a smaller 2-cell lying within the original $\sigma^{2}$, mapping this small 2-cell just as $f$ mapped $\sigma^{2}$, and mapping the region between the original boundary of $\sigma^{2}$ and the new 2-cell into $P$. Clearly, this may be done by a deformation of $f$ in $\sigma^{2}$. Proceeding in this way we deform $f$ in all the 2-cells of $S_{0}^{2}$, so that all of $S_{0}^{2}$ is mapped into $P$, except for a number of small islands, each of which is mapped either into $W$ or into $S^{2}$. We may now push all the islands which go into $W$ around $S_{0}^{2}$ so that they lie in one hemisphere, and all those which go in to $S^{2}$ so that they lie in the other, and so that the equatorial circle which separates the two classes goes through $P_{0}$ and is mapped in to $P$. The resulting mapping defines an element of $\pi_{2}(W)$ and one of $\pi_{2}\left(S^{2}\right)$, which is isomorphic with $I_{0}$. Thus, to each mapping $f$ defining an element of $\pi_{2}\left(W+S^{2}\right)$ corresponds a pair of mappings $f_{1}, f_{2}$ of $\pi_{2}(W)$ and $\pi_{2}\left(S^{2}\right)$ respectively, and this correspondence is the desired isomorphism. This completes the proof.

Now consider a mapping $f$ of $S_{0}^{2}$ in to $T=$ torus with patch, and the corresponding induced mapping $\bar{f}$ of $S_{0}^{2}$ in to $\bar{T}=$ the covering space of $T$. Since $S_{0}^{2}$ is compact, we can drop all but a finite block of fundamental regions $\bar{T}_{i j}$ from $\bar{T}$ without affecting $f$. The remaining space may be constructed by the addition of fundamental regions to each other a finite number of times, and the lemma gives the result expressed in

(13) We remark that $\pi_{2}(T)$ and $\pi_{2}(\bar{T})$ are not altered if we pull the boundary of the patches to single points, so that each becomes a 2 -sphere touching the rest of the space at a single point. 
THEOREM 1. The 2-dim. homotopy group $\pi_{2}(T)$, where $T=$ torus with patch, is isomorphic with the direct sum $\sum^{\infty} I_{0}$, where the elements of $\sum^{\infty} I_{0}$ are infinite sequences of integers, only a finite number of which differ from 0 .

To bring out clearly the effect of $G$ operating on $\pi_{2}(T)$ we may represent the elements of $G$ by ordered pairs of integers $g=\left(\alpha_{1}, \alpha_{2}\right)$, and the elements of $\pi_{2}(T)$ by matrices $h=\left\|a_{i j}\right\|$; then $g h$ is the matrix obtained from $h$ by a shift of $\alpha_{1}$ places horizontally and $\alpha_{2}$ places vertically.

An intuitive interpretation of the element $a_{i j}$ in the matrix of the element $h$ of $\pi_{2}(T)$ corresponding to the mapping $\bar{f}$ of $S_{0}^{2}$ into $\bar{T}$ may be given as follows: only a finite number of fundamental regions $\bar{T}_{i j}$ will contain images of points of $S_{0}^{2}$. Cut $\bar{T}$ along a rectangle containing all of these, and draw rays from the center of the patch $\bar{A}_{i j}$. By pushing along these rays we may alter $\bar{f}$ so that all points of $S_{0}^{2}$ go into points of the little 2 -sphere formed by the patch $\bar{A}_{i j}$ and its underlying simplex, while all points which were originally mapped into points of this 2 -sphere remain fixed. (For this purpose we may identify all patches other than $\bar{A}_{i j}$ with their underlying simplexes.) Then we have a mapping of $S_{0}^{2}$ into a 2 -sphere, and its degree in the ordinary sense will be precisely $a_{i j}$. This may be interpreted roughly as the number of times $S_{0}^{2}$ covers the small 2 -sphere, or, equivalently, as the number of times the patch $\bar{A}_{i j}$ is covered by the mapping $\bar{f}$ of $S_{0}^{2}$.

The reader may find it profitable to consider the details, similar to those we have given, for the case where $T$ is the topological product of three circles, or where the fundamental group of $T$ is isomorphic with the additive group of integers modulo $n$.

6. Normal mappings; the degree of a mapping. We return to the case of an arbitrary space $T$.

The first vertex of a cell of a complex whose vertices are ordered as in $\$ 4$ will be called its leading vertex. It is not true that any mapping of a complex $K$ into $T$ may be deformed into a standard mapping. But we may alter standard mappings on each 2-cell near its leading vertex so that the resulting class of mappings is perfectly general in the sense of homotopy. We do this as follows: let $f^{0}$ be a standard mapping of $x_{i} x_{j} x_{k}$ in to $T$. Deform $f^{0}$, keeping the boundary fixed, so that a small 2-cell inside $x_{i} x_{j} x_{k}$ and touching the boundary only at $x_{i}$ goes into $P$. Now replace $f^{0}$ on this 2 -cell by a mapping which defines an arbitrary element of $\pi_{2}(T)$. The resulting mapping of $x_{i} x_{j} x_{k}$ into $T$ will be called normal over $x_{i} x_{j} x_{k}$. If a mapping is normal over all the 2-cells of $K$ it will be called normal. The following lemma holds:

Lemмa 3. Any mapping $f$ of $K$ into $T$ may be deformed into a normal mapping.

Proof. First we may deform $f$ into a mapping normal over $K^{1}$, by Lemma 1. Now consider any 2-cell $\sigma^{2}$ of $K$. Make another copy of $\sigma^{2}$ and define on it 
the standard mapping $f^{0}$ which coincides with $f$ along the boundary of $\sigma^{2}$, altered so that a small 2 -cell near $x_{i}$ goes into $P$, as above. Join the two 2 -cells along their boundaries, and consider the resulting mapping $\phi$ of the 2 -sphere thus formed into $T$. The boundary of the small 2-cell divides the 2 -sphere into two parts, and itself goes into $P$, so that the element of $\pi_{2}(T)$ thus defined, taking $x_{i}$ as the fixed point of the 2-sphere, is the sum of two elements, one of which may be chosen at pleasure. By choosing it properly, we can make the resulting mapping homotopic to 0 . This is equivalent to saying that $\phi$ may be extended throughout the interior of the 2 -sphere, or that the mapping $f$ may be deformed into a mapping normal on $\sigma^{2}$, leaving the boundary of $\sigma^{2}$ fixed. Thus we may deform $f$ over each 2-cell of $K$ until it is normal.

The element of $\pi_{2}(T)$ by which the standard mapping $f^{0}$ must be altered to give a mapping deformable in to $f$ on $x_{i} x_{j} x_{k}$ will be called the degree of $f$ on $x_{i} x_{j} x_{k}$, and denoted by $d_{f}\left(x_{i} x_{j} x_{k}\right)$. This is simply the negative of the element of $\pi_{2}(T)$ defined by $f^{0}$ unchanged and $f$ when the boundaries are identified as above. Clearly, if $f$ is already standard on $x_{i} x_{j} x_{k}$, then $d_{f}\left(x_{i} x_{j} x_{k}\right)=0$, so that the degree of a mapping on a 2-cell is a measure of its deviation from the corresponding standard mapping.

Let $E^{3}=x_{i} x_{j} x_{k} x_{l}$ be a three-cell of $K$, and let $f$ map the boundary $\partial x_{i} x_{j} x_{k} x_{l}$ of $E^{3}$ into $T$, with the leading vertex $x_{i}$ going into $P$. Then $f$ defines an element of $\pi_{2}(T)$ if we regard $\partial E^{3}$ as a 2 -sphere with fixed point $x_{i}$. We shall denote this element of $\pi_{2}(T)$ by $D_{f}\left(\partial E^{3}\right)$ and call it the degree of $f$ on $\partial E^{3}$. Suppose $f$ is normal over $\partial E^{3}$ and $f^{0}$ is the corresponding standard mapping. We wish to find a relation between $D_{f}{ }^{0}\left(\partial E^{3}\right)$ and $D_{f}\left(\partial E^{3}\right)$. The mapping $f$ is obtained from $f^{0}$ by replacing in each 2-cell of $\partial E^{3}$ a small piece going into $P$ by one which defines an element of $\pi_{2}(T)$. The following theorem is an immediate consequence of (3.5):

THEOREM 2. If $E^{3}=x_{i} x_{j} x_{k} x_{l}$ is a 3-cell of $K$, and $f$ is a normal mapping of $\partial E^{3}$ into $T$, then

$$
\begin{aligned}
D_{f}\left(\partial E^{3}\right)= & D_{f^{0}}\left(\partial E^{3}\right)-d_{f}\left(x_{i} x_{j} x_{k}\right)+d_{f}\left(x_{i} x_{j} x_{l}\right) \\
& -d_{f}\left(x_{i} x_{k} x_{l}\right)+g\left(x_{i} x_{j}\right) d_{f}\left(x_{j} x_{k} x_{l}\right),
\end{aligned}
$$

where $g\left(x_{i} x_{j}\right)$ is the element of $G$ into which $f$ maps $x_{i} x_{j}$.

7. An extension theorem. Let $f$ be a normal mapping of the subcomplex $K^{*}$ of a simplicial 3 -complex $K$ into $T$; that is, all vertices $x_{i}$ go into $P$, all 1 -cells $x_{i} x_{j}$ into elements of $G$ in normal form, and all 2-cells of $K^{*}$ are mapped normally. We wish to find conditions that $f$ may be extended throughout all of $K$. First, when may $f$ be extended throughout all the 1- and 2-cells of $K$ ? Let $\sigma^{2}=x_{i} x_{j} x_{k}$ be a 2-cell of $K$; then if $x_{i} x_{j}, x_{j} x_{k}$, and $x_{i} x_{k}$ are mapped in to the elements $g\left(x_{i} x_{j}\right)$, etc., of $G$, the necessary and sufficient condition that this mapping may be extended throughout the interior of $\sigma^{2}$ is that $g\left(x_{i} x_{j}\right) \cdot g\left(x_{j} x_{k}\right)$ 
$\cdot g^{-1}\left(x_{i} x_{k}\right)=1$, or in other words, that running around the boundary of $\sigma^{2}$ defines the unit element of $G$. It is easily seen that neither the sense nor the initial point of the circuit matters for this purpose. Thus, if we define the coboundary $\delta A^{1}$ of the $1-G$-chain

$$
A^{1}=\sum g\left(x_{i} x_{j}\right) x_{i} x_{j}
$$

to be the $2-G$-chain

$$
A^{2}=\sum g\left(x_{i} x_{j} x_{k}\right) x_{i} x_{j} x_{k}
$$

where

$$
g\left(x_{i} x_{j} x_{k}\right)=g\left(x_{i} x_{j}\right) \cdot g\left(x_{j} x_{k}\right) \cdot g^{-1}\left(x_{i} x_{k}\right),
$$

then the necessary and sufficient condition that $f$ defined on $K^{*}$ may be extended throughout all the 2-cells of $K$ is that a $1-G$-chain $A_{1}^{1}$ of $K$ of the form (7.1) exist such that $\delta A_{1}^{1}$ is a $2-G$-chain each of whose coefficients is 1 , where $g\left(x_{i} x_{j}\right)$ is defined by $f$ on those 1-cells $x_{i} x_{j}$ which belong to $K^{*}$. A $1-G$-chain of $K$ whose coboundary vanishes in this sense will be called a cocycle; then our condition is that the $1-G$-chain

$$
A_{0}^{1}=\sum g\left(x_{i} x_{j}\right) x_{i} x_{j}
$$

summed over all $x_{i} x_{j}$ in $K^{*}$ be part of a cocycle $A_{1}^{1}$; i.e., that elements $g\left(x_{i} x_{j}\right)$ may be assigned to the 1 -cells $x_{i} x_{j}$ in $K-K^{*}$ so that the chain (7.4) then becomes a cocycle. We shall return to this matter of chains with coefficients from a non-abelian group in $\$ 9$.

Now we assume that $f$ has been defined throughout all the 2-cells of $K$; when may it be extended throughout all the 3 -cells? Let $E^{3}=x_{i} x_{j} x_{k} x_{l}$ be such a 3 -cell; regard its boundary as a 2 -sphere with fixed point $x_{i}$. Then $f$ may be extended throughout the interior of $E^{3}$ if and only if

$$
D_{f}\left(\partial E^{3}\right)=0 \text {, }
$$

and $f$ may be extended throughout all the 3-cells of $K$ if and only if $C^{3}=$ $\sum D_{f}\left(\partial E^{3}\right) E^{3}=0$, where the summation is over all 3 -cells $x_{i} x_{j} x_{k} x_{l}$ of $K$. By equation (6.1) we have

$$
\begin{aligned}
C^{3}= & \sum D_{f^{0}}\left(\partial x_{i} x_{j} x_{k} x_{l}\right) x_{i} x_{j} x_{k} x_{l}+\sum\left[-d_{f}\left(x_{i} x_{j} x_{k}\right)+d_{f}\left(x_{i} x_{j} x_{l}\right)\right. \\
& \left.-d_{f}\left(x_{i} x_{k} x_{l}\right)+g\left(x_{i} x_{j}\right) d_{f}\left(x_{j} x_{k} x_{l}\right)\right] x_{i} x_{j} x_{k} x_{l} .
\end{aligned}
$$

We shall now consider the meaning of the first term on the right side of this equation.

As usual, let $G$ be the fundamental group of $T$ and $\pi_{2}(T)$ the 2-dim. homotopy group. We shall define a new sort of operation $g_{1} \circ g_{2}$ on the elements of $G$ giving standard mappings of 2 -cells into $T$, and an operation $g_{1} \circ g_{2} \circ g_{3}$ giving elements of $\pi_{2}(T)$ : 
(a) $g_{1} \circ g_{2}$ is the standard mapping of a 2-cell $x_{i} x_{j} x_{k}$ into $T$ defined as follows: $\operatorname{map} x_{i} x_{j}$ into $g_{1}$ and $x_{j} x_{k}$ into $g_{2}$; then the uniquely determined standard mapping of $x_{i} x_{j} x_{k}$ into $T$ will be denoted by $g_{1} \circ g_{2}$.

(b) Let $\phi=g_{1} \circ g_{2}$ be a standard mapping of $x_{i} x_{j} x_{k}$ into $T$; then by $\phi \circ g_{3}=\left(g_{1} \circ g_{2}\right) \circ g_{3}$ we mean the standard mapping of $\partial x_{i} x_{j} x_{k} x_{l}$ into $T$ determined by mapping $x_{i} x_{j} x_{k}$ into $T$ by $\phi$ and $x_{k} x_{l}$ by $g_{3}$. We may omit parentheses and define $g_{1} \circ g_{2} \circ g_{3}$ to be the standard mapping of $\partial x_{i} x_{j} x_{k} x_{l}$ in to $T$ defined by mapping $x_{i} x_{j}, x_{j} x_{k}$, and $x_{k} x_{l}$ into $g_{1}, g_{2}$, and $g_{3}$ respectively. This mapping is uniquely determined. For knowing $g\left(x_{i} x_{j}\right)$ and $g\left(x_{j} x_{k}\right)$ gives $g\left(x_{i} x_{k}\right)$, defining uniquely the standard mapping on $x_{i} x_{j} x_{k}$, and likewise for the other faces of $x_{i} x_{j} x_{k} x_{l}$. Since each standard mapping of $\partial x_{i} x_{j} x_{k} x_{l}$ into $T$ determines uniquely an element of $\pi_{2}(T)$ we may regard the operation $\circ$ when applied to three elements of $G$ as giving an element of $\pi_{2}(T)\left({ }^{14}\right)$.

Now let us return to (7.6). As before, let

$$
A_{1}^{1}=\sum g\left(x_{i} x_{j}\right) x_{i} x_{j}
$$

summed over all $x_{i} x_{j}$ of $K$ be the cocycle of which $A_{0}^{1}$ is a part; then

$$
\sum D_{f^{0}}\left(\partial x_{i} x_{j} x_{k} x_{l}\right) x_{i} x_{j} x_{k} x_{l}=A_{1}^{1} \cup A_{1}^{1} \cup A_{1}^{1},
$$

where the multiplication of elements of $G$ is understood in the sense just defined.

It remains to investigate the second sum of (7.6). We may write

$$
\begin{gathered}
-d_{f}\left(x_{i} x_{j} x_{k}\right)+d_{f}\left(x_{i} x_{j} x_{l}\right)-d_{f}\left(x_{i} x_{k} x_{l}\right)+g\left(x_{i} x_{j}\right) d_{f}\left(x_{j} x_{k} x_{l}\right) \\
=-d_{f}\left(x_{i} x_{j} x_{k}\right)+d_{f}\left(x_{i} x_{j} x_{l}\right)-d_{f}\left(x_{i} x_{k} x_{l}\right)+d_{f}\left(x_{j} x_{k} x_{l}\right) \\
\quad+\left(g\left(x_{i} x_{j}\right)-1\right) d_{f}\left(x_{j} x_{k} x_{l}\right)
\end{gathered}
$$

where $\left(g\left(x_{i} x_{j}\right)-1\right)$ is an element of $\bar{G}$. Let

$$
\begin{aligned}
& A_{1}^{2}=\sum d_{f}\left(x_{i} x_{j} x_{k}\right) x_{i} x_{j} x_{k}, \\
& A_{3}^{1}=\sum\left(g\left(x_{i} x_{j}\right)-1\right) x_{i} x_{j},
\end{aligned}
$$

where the summation is over all 2- and 1-cells of $K$, respectively. Then from (7.6), (7.8), and (7.9) we have

$$
C^{3}=A_{1}^{1} \cup A_{1}^{1} \cup A_{1}^{1}+\delta A_{1}^{2}+A_{3}^{1} \cup A_{1}^{2}
$$

This proves

TheOREM 3. Let $K^{*}$ be a subcomplex of $K=K^{3}$ and let $f$ be a normal mapping

(14) Perhaps the main difficulty of the classification problem is that of determining this multiplication in any concrete case. 
of $K^{*}$ into $T$. Necessary and sufficient conditions that $f$ may be extended throughout $K$ are

(i) that $A_{0}^{1}$ be part of a cocycle $A_{1}^{1}$,

(ii) that the 2-chain $A_{1}^{2}$ with coefficients from $\pi_{2}(T)$ exist such that $A_{1}^{1} \cup A_{1}^{1} \cup A_{1}^{1}$ $+\delta A_{1}^{2}+A_{3}^{1} \cup A_{1}^{2}=0$, where

$$
A_{0}^{1}=\sum g\left(x_{i} x_{j}\right) x_{i} x_{j}
$$

summed over all $x_{i} x_{j}$ of $K^{*}$,

$$
A_{1}^{2}=\sum d_{i j k} x_{i} x_{j} x_{k}
$$

summed over all $x_{i} x_{j} x_{k}$ of $K$ and such that $d_{i j k}=d_{f}\left(x_{i} x_{j} x_{k}\right)$ whenever $x_{i} x_{j} x_{k}$ is in $K^{*}$, and

$$
A_{3}^{1}=\sum\left(g\left(x_{i} x_{j}\right)-1\right) x_{i} x_{j}=A_{1}^{1}-I^{1}
$$

where $I^{1}=\sum 1 \cdot x_{i} x_{j}$ summed over all $x_{i} x_{j}$ of $K$.

Note that in $A_{1}^{1} \cup A_{1}^{1} \cup A_{1}^{1}$ the "product" of elements of $G$ is either a standard mapping or an element of $\pi_{2}(T)$, while in $A_{3}^{1} \cup A_{1}^{2}$ the elements of $G$ are operators on $\pi_{2}(T)$.

8. The classification. Let $K$ be a 2 -complex and let $f^{1}$ and $f^{2}$ be normal mappings of $K$ into the space $T$. Let $K \times I$ be the product-complex of $K$ with the unit interval; then $f^{1}$ and $f^{2}$ are homotopic if and only if there exists a mapping $F$ of $K \times I$ into $T$ such that $F(x, 0) \equiv f^{1}(x)$ and $F(x, 1) \equiv f^{2}(x)$. In this section we shall specialize the result of $\S 7$ to the case where $K^{3}=K^{2} \times I$, and where $F$ is defined on $K \times 0+K \times 1$ by $f^{1}$ and $f^{2}$ respectively.

We may subdivide $K \times I$ into cells of the form $\sigma_{j}^{i} \times I$, where the $\sigma_{j}^{i}$ are the cells of $K$. If in particular $K$ is of the form considered in $\$ 4$, we shall orient $K \times I$ as follows: the orientation of $K \times 0$ and $K \times 1$ shall be as in $K$, with corresponding vertices $y_{i}$ and $z_{i}$. The 1 -cells $y_{i} z_{i}$ shall be positively oriented in that form. Orientation of the 2 -cells $x_{i} x_{j} \times I$ shall be such as to put $y_{i} y_{j}$ on the boundary of $x_{i} x_{j} \times I$ in the positive sense, and the oriented 3-cell $x_{i} x_{j} x_{k} \times I$ shall have $y_{i} y_{j} y_{k}$ on its boundary in the positive sense. Of course, $K \times I$ as defined is not a simplicial complex; it may be simplicially subdivided, and we shall do this later.

When may $F$ as defined above be extended throughout all the 2-cells of $K \times I$ ? The remaining 2 -cells are of the form $x_{i} x_{j} \times I$. Clearly, the necessary and sufficient condition is that elements $a_{i}$ of $G$ exist such that

$$
f_{i j}^{1} a_{j}\left(f_{i j}^{2}\right)^{-1}\left(a_{i}\right)^{-1}=1
$$

for all $i<j$ such that $x_{i} x_{j}$ is a 1-cell of $K$, and where $f_{i j}^{1}$ and $f_{i j}^{2}$ are respectively the elements of $G$ into which $f^{1}$ and $f^{2}$ map $x_{i} x_{j}$. We may write this in the form 


$$
f_{i j}^{1}=a_{i} f_{i j}^{2} a_{j}^{-1} .
$$

We shall call two $1-G$-chains $C^{1}=\sum g_{i j} x_{i} x_{j}$ and $C^{2}=\sum h_{i j} x_{i} x_{j}$ cohomologous if elements $\alpha_{i}$ of $G$ exist such that

$$
g_{i j}=\alpha_{i} h_{i j} \alpha_{j}^{-1},
$$

and write $C^{1} \backsim C^{2}$; this reduces to the ordinary definition if $G$ is abelian. It is natural to write $A_{1}^{1} / A_{2}^{1}=\delta A^{0}$ if $A_{1}^{1}$ and $A_{2}^{1}$ are so related, where

$$
A^{0}=\sum \alpha_{i} x_{i} .
$$

The 0 - $G$-chain $A^{0}$ is uniquely determined, if $K$ is connected, when the coefficient of any vertex is assigned.

We may now assert that $F$ as defined may be extended throughout all the 2 -cells of $K \times I$ if and only if

$$
A_{1}^{1} \sim A_{2}^{1} \text { or } A_{1}^{1} / A_{2}^{1}=\delta A^{0}
$$

where

$$
A_{1}^{1}=\sum f_{i j}^{1} x_{i} x_{j}, \quad A_{2}^{1}=\sum f_{i j}^{2} x_{i} x_{j}, \quad A^{0}=\sum a_{i} x_{i} .
$$

Let us now suppose the extension throughout the 2-cells $x_{i} x_{j} \times I$ made in a normal manner; when may we extend the resulting mapping throughout the 3 -cells $x_{i} x_{j} x_{k} \times I$ ? The boundary of the 3 -cell $E^{3}=x_{i} x_{j} x_{k} \times I$ may be regarded as a 2 -sphere with fixed point $y_{i}$, and the normal mapping of it defines an element $D_{F}\left(\partial E^{3}\right)$. The necessary and sufficient condition that this mapping may be extended throughout the interior of $E^{3}$ is that $D_{F}\left(\partial E^{3}\right)=0$, from which it follows that the $\mathrm{n}$. and s. condition that this be possible for all the 3-cells of $K \times I$ is that

$$
\sum D_{F}\left(\partial E^{3}\right) E^{3}=\sum D_{F}\left(\partial x_{i} x_{j} x_{k} \times I\right) x_{i} x_{j} x_{k} \times I=0 .
$$

It follows from (3.5) that

$$
\begin{aligned}
D_{F}\left(\partial E^{3}\right)= & D_{F^{0}}\left(\partial E^{3}\right)+d_{f^{1}}\left(x_{i} x_{j} x_{k}\right)-d_{F}\left(x_{i} x_{j} \times I\right) \\
& +d_{F}\left(x_{i} x_{k} \times I\right)-f_{i j}^{1} d_{F}\left(x_{j} x_{k} \times I\right)-a_{i} d_{f^{2}}\left(x_{i} x_{j} x_{k}\right) .
\end{aligned}
$$

Now let

$$
\begin{gathered}
\eta_{i j k}=d_{f^{1}}\left(x_{i} x_{j} x_{k}\right)-a_{i} d_{f^{2}}\left(x_{i} x_{j} x_{k}\right), \quad \zeta_{i j k}=D_{F^{\circ}} \partial\left(x_{i} x_{j} x_{k} \times I\right), \\
b_{i j k}=d_{F}\left(x_{i} x_{j} \times I\right) .
\end{gathered}
$$

Then

$$
D_{F}\left(\partial x_{i} x_{j} x_{k} \times I\right)=\zeta_{i j k}+\eta_{i j k}-\left[b_{i j}-b_{i k}+b_{j k}\right]-\left(f_{i j}^{1}-1\right) b_{i k},
$$

so that 


$$
\begin{aligned}
\sum D_{F}\left(\partial E^{3}\right) E^{3}= & \sum \zeta_{i j k} x_{i} x_{j} x_{k} \times I+\sum \eta_{i j k} x_{i} x_{j} x_{k} \times I \\
& -\sum\left[b_{i j}-b_{i k}+b_{j k}\right] x_{i} x_{j} x_{k} \times I \\
& -\sum\left(f_{i j}^{1}-1\right) b_{j k} x_{i} x_{j} x_{k} \times I .
\end{aligned}
$$

Now let

$$
\begin{array}{rlrl}
B^{2} & =\sum \zeta_{i j k} x_{i} x_{j} x_{k}, & B^{1}=\sum b_{i j} x_{i} x_{j}, \\
C^{2}=\sum \eta_{i j k} x_{i} x_{j} x_{k}, & \bar{A}_{1}^{1}=\sum\left(f_{i j}^{1}-1\right) x_{i} x_{j} .
\end{array}
$$

Then (8.7) becomes

$$
B^{2}+C^{2}=\delta B^{1}+\bar{A}_{1}^{1} \cup B^{1} .
$$

We shall now find a simple expression for the term $B^{2}$.

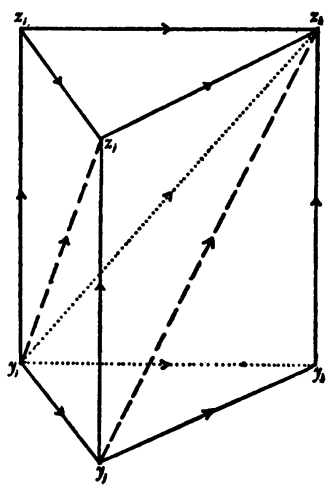

FIG. 2

We subdivide $x_{i} x_{j} x_{k} \times I$ simplicially, as shown in Figure 2. (This subdivision is easy to define combinatorially.) Then

$$
\begin{aligned}
& A_{1}^{1} \cup A_{1}^{1} \cup A^{0}=\sum\left(f_{i j}^{1} \circ f_{j k}^{1} \circ a_{k}\right) x_{i} x_{j} x_{k}, \\
& A_{1}^{1} \cup A^{0} \cup A_{2}^{1}=\sum\left(f_{i j}^{1} \circ a_{j} \circ f_{j k}^{2}\right) x_{i} x_{j} x_{k}, \\
& A^{0} \cup A_{2}^{1} \cup A_{2}^{1}=\sum\left(a_{i} \circ f_{i j}^{2} \circ f_{j k}^{2}\right) x_{i} x_{j} x_{k},
\end{aligned}
$$

and from (3.6) it follows, taking account of orientation, that

$$
B^{2}=-A_{1}^{1} \cup A_{1}^{1} \cup A^{0}+A_{1}^{1} \cup A^{0} \cup A_{2}^{1}-A^{0} \cup A_{2}^{1} \cup A_{2}^{1}
$$

Thus (8.13) becomes

$$
\begin{array}{r}
C^{2}-A_{1}^{1} \cup A_{1}^{1} \cup A^{0}+A_{1}^{1} \cup A^{0} \cup A_{2}^{1}-A^{0} \cup A_{2}^{1} \cup A_{2}^{1} \\
=\delta B^{1}+\bar{A}_{1}^{1} \cup B^{1} .
\end{array}
$$

We may now state 
THEOREM 4. A necessary and sufficient condition that the normal mappings $f^{1}$ and $f^{2}$ of $K=K^{2}$ into $T$ be homotopic is that a $0-G$-chain $A^{0}$ and a $1-\pi_{2}(T)$ chain $B^{1}$ of $K$ exist, satisfying

(i) $A_{1}^{1} / A_{2}^{1}=\delta A^{0}$,

(ii) $(8.16)$,

where

$$
\begin{gathered}
A_{1}^{1}=\sum f_{i j}^{1} x_{i} x_{j}, \quad A_{2}^{1}=\sum f_{i j}^{2} x_{i} x_{j}, \quad \bar{A}_{1}^{1}=\sum\left(f_{i j}^{1}-1\right) x_{i} x_{j}=A_{1}^{1}-I^{1}, \\
C^{2}=\sum d_{f^{1}}\left(x_{i} x_{j} x_{k}\right)-a_{i} d_{f^{2}}\left(x_{i} x_{j} x_{k}\right) x_{i} x_{j} x_{k},
\end{gathered}
$$

and where $A^{0}=\sum a_{i} x_{i}$.

In certain cases, $(8.16)$ becomes simplified, since the chain $B^{2}$ is automatically 0 ; this is so when $T=$ torus with patch, for the standard mappings of $\partial E^{3}$ into $T$ cover no patches, and are therefore of degree 0 , since the 2-dim. homotopy group of the plane vanishes. For this case, $(8.16)$ becomes simply

$$
C^{2}=\delta B^{1}+\bar{A}_{1}^{1} \cup B^{1} \text {. }
$$

This is also the case for any space with the fundamental group of the torus; of course the interpretation of the elements of $\pi_{2}(T)$ as matrices of integers will in general require modification, the integers being replaced by the elements of $\pi_{2}(T)$.

9. 1-Chains with coefficients from a non-abelian group. We shall conclude with a brief account of 1-G-chains, where $G$ is a non-abelian group, in extension of the remarks in the preceding two sections. These results were found independently by $\mathrm{H}$. Whitney.

Let $K$ be a complex as in $\S 4$. By a $1-G$-chain we mean a function $f(p, q)$ defined for all ordered pairs of integers $(p, q)$ for which $x_{p} x_{q}$ is a 1-cell of $K$, with values in $G$, and such that $f(p, q)=f^{-1}(q, p)$. We may denote such a chain by the symbol $C=\sum g_{i j} x_{i} x_{j}$, where $g_{i j}=f(i, j) . C$ is a cocycle if running around the boundary of each 2-cell of $K$ defines the unit element of $G$. The chain $C$ is a coboundary if there exist $h_{i}$ in $G$ such that

$$
g_{i j}=h_{i}^{-1} h_{j}
$$

for all $i, j$ such that $x_{i} x_{j}$ is a 1 -cell of $K$. If $C$ is a coboundary it is a cocycle. Let $C=\sum g_{i j} x_{i} x_{j}$ be a chain, and $D=\sum h_{i} x_{i}$ be a $0-G$-chain; then by $C \circ D$ we mean the chain

$$
\sum\left(h_{i}^{-1} g_{i j} h_{j}\right) x_{i} x_{j} .
$$

If $C$ is a cocycle, so is $C \circ D$; if $C$ is a coboundary, so is $C \circ D$. The operation o is associative, there is a unit, and inverses exist. If $C_{1}$ and $C_{2}$ are cocycles, and $C_{1}=C_{2} \circ D$ for some $0-G$-chain $D$, we say that $C_{1}$ and $C_{2}$ are cohomologous; in symbols, $C_{1} \backsim C_{2}$ or $C_{1} / C_{2}=\delta D$. If $G$ is abelian, this reduces to the ordinary notion of cohomology. The preceding remarks show that the set of 0 - $G$-chains 
form a group of transformations acting on the set of 1-G-cocycles. We shall call the class of all cocycles which are homologous to $C$ the coset of $C$, and denote it by $[C]$. Two cosets are identical or disjoint. A chain will be called part of a cocycle if some or all of its coefficients which are 1 's may be replaced by other elements of $G$ so that the resulting chain is a cocycle.

Let $f^{1}$ and $f^{2}$ be two mappings of a complex $K$ into an aspherical( $\left.{ }^{15}\right)$ space $T$ with fundamental group $G$. They are homotopic if and only if the mapping $F$ defined on $K \times 0+K \times 1$ by them may be extended throughout the 2 -cells $x_{k} x_{j} \times 1$ of $K \times 1$; for since $T$ is aspherical, it may then be extended throughout all the 3-, 4-, $\cdots$ cells. This is possible if and only if the 1-G-chain $C_{f^{1}, f^{2}}$ with coefficients $f_{i j}^{1}$ on $y_{i} y_{j}$ and $f_{i j}^{2}$ on $z_{i} z_{j}$ is part of a cocycle. But this is so if and only if the 1-G-cocycles $C_{f^{1}}=\sum f_{i j}^{1} x_{i} x_{j}$ and $C_{f^{2}}=\sum f_{i j}^{2} x_{i} x_{j}$ of $K$ are cohomologous. Thus we have

THEOREM 5. The classes of mappings of $K$ into the aspherical space $T$ are in one-to-one correspondence with the cosets $[C]$, where the $C$ are the 1-G-cocycles of $K$, and $G$ is the fundamental group of $T$.

This theorem is equivalent to the well known theorem of Brouwer and Hurewicz which states that the classes of mappings of $K$ into $T$ are in one-toone correspondence with the homomorphism-classes of $H=$ fundamental group of $K$ into $G=$ fundamental group of $T$, since the correspondence $\psi_{f} \leftrightarrow\left[C_{f}\right]$, where $\psi_{f}$ denotes the class of homomorphisms derived from that induced by the mapping $f$ under the set of inner automorphisms of $G$, and $C_{f}=\sum f_{i j} x_{i} x_{j}$, where $f_{i j}$ is the element of $G$ into which $x_{i} x_{j}$ is mapped by $f$, is easily seen to be one-to-one. (We assume $f$ to be normal; see $\S 6$.) $C_{f} \backsim C_{g}$.

Proof. We must show that (a) if $C_{f} \backsim C_{g}$ then $\psi_{f}=\psi_{g}$, (b) if $\psi_{f}=\psi_{g}$ then

We shall use the vertex $x_{0}$ as a fixed point in defining the fundamental group.

Ad (a): Run around any circuit in $K$ by $f$, getting $f_{01} f_{12} \cdots f_{p 0}$ of $G$. Then run around the circuit by $g$, getting, since $g_{i j}=h_{i}^{-1} f_{i j} h_{j}$,

$$
\left(h_{0}^{-1} f_{01} h_{1}\right)\left(h_{1}^{-1} f_{12} h_{2}\right) \cdots\left(h_{p}^{-1} f_{p 0} h_{0}\right)=h_{0}^{-1}\left(f_{01} f_{12} \cdots f_{p 0}\right) h_{0} .
$$

Ad (b): Say a closed path $C$ from $x_{0}$ in $K$ mapped by $g$ into the element $\beta(C)$ of $G$ is mapped by $f$ into $\alpha^{-1} \beta(C) \alpha$. Join each vertex $x_{i}$ to $x_{0}$ by a path $C_{i}$, and let $h_{i}=g^{-1}\left(C_{i}\right) \alpha f\left(C_{i}\right)$. Then $f\left(C_{i}\right) f_{i j} f^{-1}\left(C_{j}\right)=\alpha^{-1} g\left(C_{i}\right) g_{i j} g^{-1}\left(C_{j}\right) \alpha$ for all $i, j$ such that $x_{i} x_{j}$ is a 1 -cell of $K$. It follows that

$$
f_{i j}=f^{-1}\left(C_{i}\right) \alpha^{-1} g\left(C_{i}\right) g_{i j} g^{-1}\left(C_{j}\right) \alpha f\left(C_{j}\right)=\left[g^{-1}\left(C_{i}\right) \alpha f\left(C_{i}\right)\right]^{-1} g_{i j}\left[g^{-1}\left(C_{j}\right) \alpha f\left(C_{j}\right)\right],
$$

so that $C_{f} \backsim C_{g}$.

(15) See Hurewicz, loc. cit., in note 3.

NeW York UNIVERSITY, New York, N. Y. 